\title{
Prevalence of Extraplacental Anastomoses in Monochorionic Twin Pregnancies
}

\author{
Ryo Yamamoto Kentaro Nakanishi Haruna Kawaguchi \\ Shusaku Hayashi Keisuke Ishii \\ Department of Maternal Fetal Medicine, Osaka Women's and Children's Hospital, Osaka, Japan
}

\section{Keywords}

Monochorionic twins - Twin-twin transfusion syndrome •

Fetoscopic laser photocoagulation · Intertwin anastomosis

\begin{abstract}
Objectives: To evaluate the prevalence of extraplacental anastomoses (EPA) and the factors associated. Methods: A retrospective study including 144 placentas from monochorionic diamniotic (MCDA) twins delivering at one institution was performed. EPA were defined as any intertwin anastomosis located outside the chorionic plate on macroscopic inspection. The association with perinatal factors and with umbilical cord insertion site was analyzed. Results: The prevalence of EPA was $4.9 \%$ (7/144). Velamentous cord insertion of both twins was significantly associated with the presence of EPA in the multivariate logistic regression model $(p=0.045)$. Discussion: EPA was found in $\approx 5 \%$ of MCDA twin pregnancies.
\end{abstract}

(c) 2020 S. Karger AG, Basel

\section{Introduction}

Twin-twin transfusion syndrome (TTTS) is the most critical complication during the perinatal course of monochorionic (MC) twin pregnancies, particularly in

karger@karger.com

(c) 2020 S. Karger AG, Basel

www.karger.com/fdt

Karger ${ }^{\prime}=$ severe cases that show high mortality and morbidity in the second trimester, if prenatal surgical treatment is not provided $[1,2]$. Fetoscopic laser photocoagulation (FLP) of the chorionic communicating vessels between both twins is an alternative for management of TTTS, as it markedly improves the prognosis in both twins [3, 4].

In the FLP procedure, the coagulation of intertwin anastomoses is performed under direct visualization via the intrauterine fetoscope using a laser fiber. For most cases, the vascular equator, that is, the hemodynamic border between MC twins, is located on the placental parenchyma. Therefore, inspection of the placental surface and coagulation using a laser is sufficient. However, in rare cases, some of the chorionic vessels branching from the cord insertion run partly or entirely on the fetal membrane and form anastomoses outside the placental parenchyma. Based on the principle that the extent of laser coagulation required during fetoscopic procedure is determined according to the edge of the placental parenchyma, anastomoses located on the outside of the placental parenchyma could contribute to the residual anastomoses after FLP owing to difficulty in detection. Residual anastomoses might result in postoperative complications, including persistent TTTS and iatrogenic twin anemia-polycythemia se- 


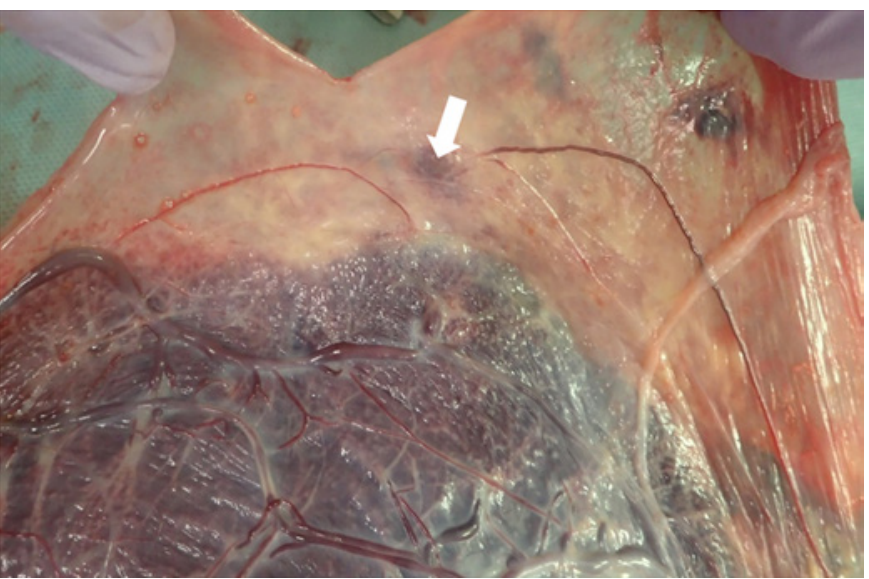

Fig. 1. Extraplacental anastomoses. Placental cord insertion sites of both twins were velamentous insertions. Each chorionic vessel that branched from the umbilical cord vessel of each twin ran entirely outside of the placental parenchyma and composed extraplacental anastomoses (arrow).

quence, which increase the mortality and morbidity in twins $[5,6]$. The objective of this study was to elucidate the prevalence of and examine the relative factors of extraplacental anastomoses (EPA).

\section{Materials and Methods}

We conducted a retrospective study on monochorionic diamniotic (MCDA) twins who were delivered at Osaka Women's and Children's Hospital, a tertiary perinatal care center in Japan, and whose placentas were inspected prospectively during the postnatal period between April 2015 and September 2019. Cases that underwent FLP or underwent either fetal demise or twin reversed arterial perfusion sequence were excluded owing to the difficulty in macroscopic inspection of the anastomoses. In our center, FLP was performed in patients with TTTS that occurred between 16 and 27 weeks of gestation and selective intrauterine growth restriction (sIUGR) accompanied by oligohydramnios and abnormal umbilical artery Doppler waveforms, as previously described [7]. All mothers provided written informed consent for data usage. The research was conducted ethically in accordance with the World Medical Association Declaration of Helsinki.

All placentas of MCDA twins were macroscopically inspected by maternal fetal medicine specialists, including at least 1 wellexperienced FLP surgeon (R.Y. or K.I.). Placentas were stored in the refrigerator until they were inspected within a few days after the delivery. The type of cord insertions was observed and classified as velamentous cord insertion (VCI), marginal cord insertion (MCI), and central cord insertion (CCI). These were defined as follows: VCI, when the vessels of the umbilical cord were inserted completely on the fetal membrane outside the placental parenchy$\mathrm{ma}$; MCI, when the umbilical vessels were inserted at the edge of the placental parenchyma with no chorionic vessels coursing to-

Extraplacental Anastomoses in

Monochorionic Twins ward the placental edge from the area of cord insertion; and CCI, cord insertion that was not classified as either VCI or MCI. Intertwin anastomoses and vascular equators were grossly examined; subsequently, as little as $10-20 \mathrm{~mL}$ of air was injected into the chorionic arteries of each twin to confirm the anastomoses. As necessary, the placental dye injection test was performed for the arteries and veins of each twin [8], and the anastomoses were classified as arterioarterial, arteriovenous, and venovenous.

The primary study outcome was the prevalence of cases with EPA. An anastomosis located outside the placental parenchyma on postnatal inspection was defined as EPA (Fig. 1). This meant that chorionic vessels that comprised the EPA could be located completely outside the placental parenchyma or partly on the placental parenchyma. Although the data on the characteristics of pregnancy were collected retrospectively, data regarding the gross examination of the placenta were collected prospectively.

The association between EPA and perinatal factors, including the site of placental cord insertion, was examined using univariate analysis. Nominal variables were compared using Fisher's exact test, while continuous variables were compared using the Wilcoxon rank-sum test. Perinatal factors associated with EPA were analyzed using a multivariate logistic regression model. $p<0.05$ was considered as statistically significant. All statistical analyses were performed using JMP version 12 (SAS Institute, Cary, NC, USA).

\section{Results}

One-hundred and eighty-two MCDA twin pregnancies met the inclusion criteria. Thirty-three cases that underwent FLP and 5 cases of fetal demise were excluded, and eventually 144 cases were included in the study analysis.

Pregnancy characteristics are listed in Table 1. The prevalence of blastocyst transfer was significantly higher among patients with EPA. The prevalence of EPA was $4.9 \%$ ( 7 cases). In terms of placental cord insertion site, the number of cases with EPA was 3 in 59 cases with CCI of both twins, 1 in 47 cases with CCI - MCI, 1 in 23 cases with CCI - VCI, and 2 in 6 cases with VCI of both twins, respectively. No EPA was found in 5 cases with MCI of both twins and in 4 cases with MCI - VCI. In 2 cases with VCI of both twins, each chorionic vessel that branched from the umbilical cord vessel of each twin ran entirely outside the placental parenchyma and constituted EPA.

Regarding the association between EPA and perinatal factors, blastocyst transfer and presence of VCI in both twins were related to the development of EPA ( $p=0.048$ and 0.027 , respectively). In the multivariate logistic regression model, only VCI in both twins was related to the development of EPA ( $p=0.045$, adjusted odds ratio $=8.25)$. 
Table 1. Characteristics and perinatal outcomes of cases with and without EPA

\begin{tabular}{lccc}
\hline & $\begin{array}{l}\text { Cases with EPA } \\
(n=7)\end{array}$ & $\begin{array}{l}\text { Cases without EPA } \\
(n=137)\end{array}$ & $p$ value \\
\hline Maternal age $\geq 35$ years & $5(71)$ & $55(40)$ & 0.12 \\
Primiparity & $4(57)$ & $70(51)$ & 1.00 \\
In vitro fertilization & $3(42)$ & $25(18)$ & 0.13 \\
$\quad$ Intracytoplasmic sperm injection & 0 & $8(5.8)$ & 1.00 \\
$\quad$ Frozen embryo transfer & $2(28)$ & $20(14)$ & 0.29 \\
$\quad$ Blastocyst transfer & $3(42)$ & $16(11)$ & 0.048 \\
Uterine myoma & $1(14)$ & $6(4.3)$ & 0.29 \\
sIUGR & $2(28)$ & $53(38)$ & 0.70 \\
Gestational age at birth, weeks & $34(33-38)$ & $70(24-39)$ & 0.13 \\
Cesarean section & $6(85)$ & $2.270(456-3.542)$ & 0.11 \\
Birth weight, g & $2.138(1.474-2.880)$ & $12(0-67)$ & 0.39 \\
Birth weight discordance, $\%$ & $12(3-25)$ & & 0.83 \\
\hline
\end{tabular}

Data are shown in $n$ (\%) or median (range). EPA, extraplacental anastomoses; sIUGR, selective intrauterine growth restriction.

\section{Discussion}

The present study demonstrated that EPA showed a prevalence of $4.9 \%$ among MCDA twin pregnancies. The related factor of EPA was VCI of both twins.

In singleton pregnancies, chorionic vessels might course along the outside of the placental parenchyma. These are termed membranous fetal vessels, such as vasa previa, which might be vulnerable to injuries; however, the course of development of membranous fetal vessels is not clearly understood. While the prevalence of the membranous fetal vessels is unknown, it is estimated to be higher than that of vasa previa ( 0.60 per 1,000 pregnancies) [9]. This was because some membranous fetal vessels that were not associated with vasa previa could be located distantly from the internal cervical os. To the best of our knowledge, the present study is the first to reveal the prevalence of EPA in MCDA twins.

The association between VCI of both twins and the presence of EPA was described for the first time in our study. According to our results, the prevalence of VCI in at least 1 cord and of VCI in both cords was $23 \%(33 / 144)$ and $4.2 \%(6 / 144)$, respectively, which was comparable to the findings of a previous report from our center [10]. In the case of an MCDA twin with VCI of both fetuses, both chorionic vessels were composed of the EPA that originated outside the placental parenchyma; thereafter, the complete vessels were located outside the placental parenchyma. It was theoretically convincing that EPA was readily formed. However, EPA was also found in 5 cases with CCI of at least 1 fetus. In these 5 cases, the large part of the chorionic vessel of at least 1 fetus that developed EPA was located on the placental parenchyma. These types of EPA might not be difficult to identify during FLP because it seemed possible to find the vessels encompassing the EPA on the placental parenchyma during vascular equator inspection and to follow the course to detect EPA. However, in cases with VCI in both twins, all the chorionic vessels that constructed the EPA were located outside the placental parenchyma; therefore, it was possible to miss the anastomosis that lay outside the placenta if the usual fetoscopic inspection was performed from the placental edge to edge. In short, this type of EPA had the risk of clinical significance in $\mathrm{MC}$ twins with disorders that FLP could be adapted.

Considering the association between VCI of both twins and EPA, preoperative detailed ultrasonographic examination of placental cord insertion site could be valuable for prediction of EPA that was difficult to be found. If chorionic vessels that extended for a long distance outside of the placental parenchyma were observed by color Doppler imaging, it might be desirable to carefully inspect the outside of the placental parenchymal edge during FLP.

The strength of the present study was that the inspection of EPA was performed prospectively by experienced maternal fetal medicine specialists. A limitation of the present study was that the prevalence of EPA was elucidated only in cases without TTTS; therefore, the prevalence of EPA in cases of TTTS that required FLP, which
Yamamoto/Nakanishi/Kawaguchi/ Hayashi/Ishii 
seemed to be important clinically, is still unclear. However, the incidence of TTTS was reported to not be influenced by the type of cord insertion $[10,11]$ and the association between VCI of both twins, and the existence of EPA would be expected to be adapted to TTTS cases as well. In a recent large series, it was reported that the occurrence of VCI in 1 or both twins increased the risk of TTTS [12]. In this study, placental cord insertion was determined prenatally, in contrast to that in previous studies. This could have been an advantage as it reduced the selection bias because cases of fetal demise that were inappropriate for postnatal examination would have been excluded in previous studies. However, prenatal determination of placental cord insertion is likely to be different from postnatal confirmation by gross examination of placenta. Although the prevalence of EPA might be higher in TTTS cases, we believe that the association between VCI and TTTS is inconclusive. Second, this study was performed in a tertiary care center, where cases of complicated MC twin gestation would be encountered more frequently than those in the general population. The prevalence of cases with VCI in both twins was reported as $1 \%$ [12]; however, the prevalence in our study was $4 \%$. Furthermore, the prevalence of sIUGR (38\%) was higher than that of the MC twin cohort.

In conclusion, inspection of the placenta during FLP is recommended owing to the presence of anastomoses that are located outside the placental parenchyma and are hard to detect, considering EPA have been found in $\approx 5 \%$ of MCDA twin pregnancies. Moreover, it might be useful for prediction of the presence of EPA to understand umbilical cord insertion of both twins preoperatively.

\section{Statement of Ethics}

The paper is exempt from ethical committee approval because all data were acquired in routine clinical practice. Written informed consent was obtained from all study subjects. The research was conducted ethically in accordance with the World Medical Association Declaration of Helsinki.

\section{Conflict of Interest Statement}

The authors have no conflicts of interest to declare.

\section{Funding Sources}

The authors did not receive any funding.

\section{Author Contributions}

Ryo Yamamoto: study conception, data collection and analysis, and writing of the manuscript. Kentaro Nakanishi, Haruna Kawaguchi, and Shusaku Hayashi: data acquisition. Keisuke Ishii: drafting and revising the work.

\section{References}

1 Mahony BS, Petty CN, Nyberg DA, Luthy DA, Hickok DE, Hirsch JH. The "stuck twin" phenomenon: ultrasonographic findings, pregnancy outcome, and management with serial amniocenteses. Am J Obstet Gynecol. 1990 Nov; $163(5$ Pt 1):1513-22.

2 Saunders NJ, Snijders RJ, Nicolaides KH. Therapeutic amniocentesis in twin-twin transfusion syndrome appearing in the second trimester of pregnancy. Am J Obstet Gynecol. 1992 Mar;166(3):820-4.

3 Senat MV, Deprest J, Boulvain M, Paupe A, Winer N, Ville Y. Endoscopic laser surgery versus serial amnioreduction for severe twinto-twin transfusion syndrome. N Engl J Med. $2004 \mathrm{Jul}$ 8;351(2):136-44.

4 Sago H, Ishii K, Sugibayashi R, Ozawa K, Sumie M, Wada S. Fetoscopic laser photocoagulation for twin-twin transfusion syndrome. J Obstet Gynaecol Res. 2018 May; 44(5):831-9.
5 Robyr R, Lewi L, Salomon LJ, Yamamoto M, Bernard JP, Deprest J, et al. Prevalence and management of late fetal complications following successful selective laser coagulation of chorionic plate anastomoses in twin-totwin transfusion syndrome. Am J Obstet Gynecol. 2006 Mar;194(3):796-803.

6 Chmait RH, Korst LM, Llanes A, Mullin P, Lee RH, Ouzounian JG. Perioperative characteristics associated with preterm birth in twin-twin transfusion syndrome treated by laser surgery. Am J Obstet Gynecol. 2013 Sep; 209(3):264-8.

7 Ishii K, Nakata M, Wada S, Murakoshi T, Sago H. Feasibility and preliminary outcomes of fetoscopic laser photocoagulation for monochorionic twin gestation with selective intrauterine growth restriction accompanied by severe oligohydramnios. J Obstet Gynaecol Res. 2015 Nov;41(11):1732-7.

8 Lewi L, Deprest J, Hecher K. The vascular anastomoses in monochorionic twin pregnancies and their clinical consequences. Am J Obstet Gynecol. 2013 Jan;208(1):19-30.
9 Ruiter L, Kok N, Limpens J, Derks JB, de Graaf IM, Mol B, et al. Incidence of and risk indicators for vasa praevia: a systematic review. BJOG. 2016 Jul;123(8):1278-87.

10 Yonetani N, Ishii K, Kawamura H, Mabuchi A, Hayashi S, Mitsuda N. Significance of velamentous cord insertion for twin-twin transfusion syndrome. Fetal Diagn Ther. 2015;38(4): 276-81.

11 Kalafat E, Thilaganathan B, Papageorghiou A, Bhide A, Khalil A. Significance of placental cord insertion site in twin pregnancy. Ultrasound Obstet Gynecol. 2018 Sep;52(3):37884.

12 Couck I, Mourad Tawfic N, Deprest J, De Catte L, Devlieger R, Lewi L. Does site of cord insertion increase risk of adverse outcome, twin-to-twin transfusion syndrome and discordant growth in monochorionic twin pregnancy? Ultrasound Obstet Gynecol. 2018 Sep; 52(3):385-9.
Extraplacental Anastomoses in Monochorionic Twins
Fetal Diagn Ther 2021;48:24-27 DOI: $10.1159 / 000510636$ 